\title{
Advances in unit commitment research under the considerations of new energy and grid security
}

\author{
Hu Bo ${ }^{1}$ Wang Shuang ${ }^{2}$ \\ ${ }^{1}$ State Grid Energy Research Institute, Beijing 100052, China \\ ${ }^{2}$ Electric Power Research Institute, Beijing 100192, China
}

\begin{abstract}
To adapt the low-carbon and green development of electric power industry, and improve the utilization efficiency of wind power and solar power, etc., it's urgent to build a system of optimizing planning and production simulation technology that incorporated with the variation of wind power and solar power and the enlarging power grid. This work presented the advance in the production simulation technology, focusing on the unit commitment (UC) research under the considerations of new energy and grid security.
\end{abstract}

Keywords: wind power, solar power, grid security, unit commitment

\section{Introduction}

The main rends of energy development in China are low-carbon and green exploitation, and the primary method is to adjust the structure of energy used to generating electric power. To adapt the low-carbon and green development of electric power industry, and improve the utilization efficiency of wind power and solar power, etc., it's urgent to build a system of optimizing planning and production simulation technology that incorporated with the variation of wind power and solar power and the enlarging power grid ${ }^{[1-5]}$.

The following content of this paper contains four sections, including 1) ad- vances in the describing technology of power prediction of new energy, and the scenario decreasing technology; 2) advances in the modeling technology of security-constrained unit commitment (SCUC) that considers new energy resources; 3) advances in the algorithm for solving the SCUC models; 4) conclusions of the concerned advances.

\section{Describing technology of power prediction of new energy, and the scenario decreasing technology}

How to incorporate the generating power prediction information of wind power and solar power into the UC modeling is an exactly new research item. At present, in the researches in China and abroad, the general technology adopted was arranging the unit commitment scheme foundationally according to the determinate generating power prediction curves.

At present, the research about the incorporation of interval prediction and probability density prediction information into the modeling and computation of SCUC models is still rare, and the main problem lies in the progress of the decreasing technology of wind power prediction scenario.

In the study of power prediction of wind power and solar power, the determinative generating power prediction is general, and also the technology of determinate prediction is mature, but with 
low precision. As the trends, interval prediction and probability density prediction are becoming the focus of researches. Those three types of prediction information can be formulates as below ${ }^{[1,2]}$.

$$
\begin{aligned}
& \left\{P_{\mathrm{ne}}^{\mathrm{fcst}}\right\}, t=1,2, \cdots, T \\
& \left\{P_{\mathrm{ne}}^{\mathrm{fcst}},\left(\underline{P}_{\mathrm{ne}}^{\mathrm{fcst}}, \bar{P}_{\mathrm{ne}}^{\mathrm{fcst}}\right)\right\}, t=1,2, \cdots, T \\
& \left\{P_{\mathrm{ne}}^{\mathrm{fcst}},\left\{\left(P_{\mathrm{ne} \alpha_{1}}, \alpha_{1}\right),\left(P_{\mathrm{ne} \alpha_{2}}, \alpha_{2}\right), \cdots\left(P_{\mathrm{ne} \alpha_{\mathrm{n}}}, \alpha_{n}\right)\right\}\right. \\
& t=1,2, \cdots, T
\end{aligned}
$$

Where, $P_{\text {ne }}^{\text {fcst }}$ stands for the point prediction value of wind power and solar power at the second $t ;\left(\underline{P}_{\mathrm{ne}}^{\mathrm{fcst}}, \bar{P}_{\mathrm{ne}}^{\text {fcst }}\right)$ is the $100 \%$ prediction interval of wind power and solar power at the second $t$ based on empirical distribution (under the consideration of the requirements to high reliability for system operation), and $\underline{P}_{\mathrm{ne}}^{\mathrm{fcst}}, \bar{P}_{\mathrm{ne}}^{\text {fcst }}$ are the lower limitation, upper limitation of the interval, respectively; $P_{\text {ne } \alpha_{i}}$ stands for the power value of scenario $i$ at the second $t, \alpha_{i}$ is the occurent probability of scenario $i$.

To lighten the computation burden for solving the SCUC models, it's necessary to decrease the number of power prediction scenario. Although the larger number of scenario could bring the higher precision of computation, also the higher number leads to longer computation time. But if the number of scenario is too low, it's difficult to ensure the precision of simulation results because of the poor incorporation of the random characteristic of wind power and solar power. So it's essential for us to utilize proper technology to decrease the number of power prediction scenario.

As the present literature have mentioned, there exists some kinds of method to the goal that using a small quantity of power prediction scenario to approximate the initial scenario with satisfactory quality. In those methods, the Probability Met- ric ${ }^{[3]}$ is one type with clear physical meaning. The basic idea of Probability Metric is applying the vector norm of scenario as the estimation value for combination of scenario with close distance of vector norm till the number of the remaining scenario is satisfactory.

\section{Modeling technology of SCUC that considers new energy resources}

The researches of UC problems began in the nineteen forties, and have quite great progress with the advances in optimization theory and computer technology. The general UC models regard the power grid as one electric point equivalently which connected with all the generating units, and the losses of power grid are negligible. In the general models, the constraints primarily are total cost of operation, system concerned and units concerned. The total cost of operation mainly includes fuel cost and start-stop cost of units, sometimes incorporates with the maintenance cost. The constraints mainly includes balance of system active power, spinning reserve requirements, minimal start and stop time of units, active power of units, status of units and units ramping constraints. Besides those general constraints, the environment constraints (emission restriction) could be incorporated into the models if need ${ }^{[4,5]}$.

The general models of UC problems cannot handle the gird constraints, so the generation plan computed from UC models often can't meet the basic requirements of safety operation of power grid. The study on SCUC problems is becoming the focus recent years. The constraints of grid security includes capacity of transmission lines, balance of system reactive power, the lower and upper limitation of reactive power of units, voltage magnitude constraints of system electric points, etc. 
In the part of SCUC modeling, the researches in China and abroad have showed the trends that considering the grid security constraints, emission restriction, system reserves and new energy resources as a whole. The most sophisticated modeling technology incorporates the determinate, interval and probability density generating power prediction information into the models to describe the uncertainty of wind power and solar power.

The objective function of SCUC with consideration of new energy is minimizing the total operation cost of system in different uncertain scenario which includes the fuel cost and start-stop cost of units. The built model is a large-scale nonlinear mixed integer planning problem which can be formulated as below:

$$
\begin{aligned}
\min & \sum_{t=1}^{T} \sum_{i=1}^{N}\left(\mu_{i t} C_{u i}+v_{i t} C_{d i}\right)+\sum_{t=1}^{T} \sum_{i=1}^{N}\left(\kappa_{i t} F_{i t}\right)+ \\
& \sum_{t=1}^{T} \sum_{i=1}^{R}\left(P_{\text {curtail_ } i_{-} t} \Delta T C_{\text {curtail }}\right)+ \\
& \sum_{k=1}^{n_{k}} \alpha_{k}\left[\sum_{t=1}^{T} \sum_{i=1}^{N}\left(\kappa_{i t} F_{i t_{-} k}\right)+\right. \\
& \left.\sum_{t=1}^{T} \sum_{i=1}^{R}\left(P_{\text {curtail_ } i_{-} t_{-} k} \Delta T C_{\text {curtail }}\right)\right]
\end{aligned}
$$

Where, $\mathrm{N}$ is the number of conventional units; $\mu_{i t}$ and $v_{i t}$ is 0-1 integer variables, stand for the unit status shifts of start and stop, $\mu_{i t}=1$ stands for the status shift from stop to start of unit $i$ at second $t$, $v_{i t}=1$ stands for the status shift from start to stop of unit $i$ at second $t ; C_{u i}$ is the start cost of unit $i ; C_{d i}$ is the stop cost of unit $i$. As besides, in formula (4), the curtailments of new energy are incorporated into the objective function as the slack variables to research the economical curtailments of wind power and solar power which can increase the economic value of system operation and enlarge the range of feasible resolution for UC problems.

In formula (4), $\kappa_{i t}$ is $0-1$ integer variable which stands for the start-stop status of units (1-start, 0-stop); $F_{i t}$ stands for the generating cost of unit $i$ at second $t$ under the determinate generating power prediction of wind power and solar power; $\mathrm{R}$ is the layout region of wind power and solar power units which can be divided by new energy resources or by influx of new energy power; $P_{\text {curtail }{ }_{-} i_{-}}$is the curtailment capacity of wind power and solar power in region $i$ at second $t ; \Delta T$ is the time interval of dispatching; $C_{\text {curtail }}$ stands for the cost of new energy curtailments; $n_{k}$ is the number of power prediction scenario; $F_{i t_{-} k}$ stands for the generating cost of unit $i$ at second $t$ under the prediction scenario $k ; P_{\text {curtail } i_{-} t_{-} k}$ is the curtailment capacity of wind power and solar power in region $i$ at second $t$ under the prediction scenario $k$.

\section{Algorithm for solving the SCUC models}

The SCUC models with considerations of new energy are very difficult to solve. To split the difference between computation efficiency and precision, there're two type of idea to solve the SCUC problems, one is modeling simplification, and the other one is computation decomposition.

The method of simplifying the models is often applying the DC flows to replace the AC flows. The start point of this method is that UC problems mainly consider the active power of units, and the flow power in the grid is primary the active power, so it's theoretical correct to replace the AC flows with DC flows with the assumption that the reactive power reserves are abundant. But this method is just an approximate technique, so the resolution may have deviation for satisfying 
the grid security. To make up the shortage of the mentioned technique, researches have inducted the two-order network model which could approximate the AC flows with higher precision, and also could consider the active power losses of transmission lines.

The method of decomposing the computation is mainly the Benders algorithm and its derivative method. The basic idea of this method is decomposing the SCUC into conventional UC problem and security constrained check. The step of security constrained check is the judgement condition of loop out the computation which be called indirect method with low constringency performance. Some researchers proposed that add the flow constraints based on DC flows as the form of Lagrange multiplier into objective function which be called direct method, so the grid security constraints could be satisfied along the computation process. Compared with the indirect method, direct method has better contingency performance and optimization effect, but because of the incorporation of Lagrange multipliers, the scale of problem is enlarged.

About the bottom algorithm of SCUC problem, the traditional algorithms are the complete enumeration method, priority method, dynamic programming method, but the computation efficiency and precision of the algorithms mentioned above can't meet the requirements of large-scale SCUC problems. Lagrange relaxation method, branch and bound method, interior point method and artificial intelligent method are promising, but still have a lot work to ensure the computation efficiency and precision of largescale SCUC problems.

\section{Conclusions}

With the development of wind power and solar power, and the interconnection of power grid, how to incorporate the variation of new energy and the gird security constraints into the UC problems becomes the research focus.

From the present research literature, some advances have been reached in the fields of describing technology of power prediction of new energy, the scenario decreasing technology, the modeling technology of SCUC that considers new energy resources and the algorithm for solving the SCUC models.

For the next step, still a lot of work need to do in the key fields of scenario decreasing technology and the algorithm for solving the SCUC models.

\section{References}

[1] A. Tuohy, P. Meibom and E. Denny, et al., "Unit commitment for systems with significant wind penetration," IEEE trans. On Power Systems, pp. 592-601, 2009.

[2] J. H. Wang, M. Shahidehpour and Z. Y. Li, "Security-constraint unit commitment with volatile wind power generation," IEEE trans. On Power Systems, pp. 1319-1327, 2008.

[3] L. Wu, M. Shahidehpour and T. Li, "Stochastic security-cinstrainted unit commitment," IEEE trans. On Power Systems, pp. 800-811, 2007.

[4] A. I. Cohen and V. R. Sherkat, "Optimization-based methods for operations scheduling," Proc. Of the IEEE, pp. 1574-1591, 1987.

[5] G. B. Sheble and G. N. Fahd, "Unit commitment literatures synopsis," IEEE trans. On Power Systems, pp. 128-135, 1994. 